MATEC Web of Conferences 24,04002 (2015)

DOI: $10.1051 /$ matec conf/ 20152404002

(c) Owned by the authors, published by EDP Sciences, 2015

\title{
Modal parameters identification and monitoring of two arches
}

\author{
Marco Belloli ${ }^{1}$, Ilmas Bayati ${ }^{1}$, Stefano Giappino ${ }^{1}$, Sara Muggiasca ${ }^{1,}$ a and Lorenzo Rosa ${ }^{1}$ \\ ${ }^{1}$ Politecnico di Milano, Department of Mechanical Engineering, 20156, Milano, Italy
}

\begin{abstract}
The paper presents the results of the modal parameters identification and of the continuous monitoring of two arches built in the new area of Expo 2015 in Milan. The activities on the arches were performed during the erection stage and they were planned as a consequence of preliminary studies performed at Politecnico di Milano wind tunnel, that highlighted dynamic instability due to the wind. In particular, the first two bending modes of the structures showed a critical behaviour and for this reason a TMD (Tuned Mass Damping) system was designed in order to control these modes. At first, frequencies, damping and modal deflected shapes were evaluated in order to check the numerical FEM model, to tune the TMD system and to check its correct functioning. The two arches were then monitored for several months to observe their dynamic behaviour under different wind conditions. A good database about the strongest and the most frequent winds in the site was obtained. The accelerations registered under strong wind conditions did not reach dangerous levels for the structures, moreover these results showed a good agreement with the wind tunnel ones.
\end{abstract}

\section{Introduction}

In the last few years, the use of slender and flexible structures in civil constructions highly increased together with the need of an aerodynamic design of this structures, due to their sensitivity to interact with the wind and to induce potential dangerous phenomena, such as vortex shedding or dynamic instability (e.g. galloping) [1, 2, 3]. For this reason, wind tunnel tests on scaled models are often required to check structures behaviour [3] and the effect of their interaction with the surrounding. Once aerodynamic problems are highlighted during wind tunnel tests, it would be necessary to design damping system to control undesirable vibrations. In several cases, TMD (Tuned mass damping) devices can be effective in increasing structural damping $[4,5]$. In order to identify the frequencies and the modal shapes of a real structure FEM model could be not sufficiently reliable, especially in the TMD tuning procedure, since the target frequencies of the structure should be known precisely. Moreover, FEM model does not permit to identify natural damping of a structure. For these reasons, tests on the real structure can be required to characterize it in terms of its modal parameters before and after the TMD installation $[6,7$, 8]. Thanks to the advance in the stand-alone acquisition systems, it is also possible to easily check the structure behaviour during a wide time-window $[9,10,11,12]$.

\footnotetext{
${ }^{\mathrm{a}}$ Corresponding author: sara.muggiasca@polimi.it
}

The present paper deals with modal parameters identification and continuous monitoring of two arches built in the new area of Expo 2015 in Milan. The two arches are disposed on the either sides of a viaduct with an aesthetical role and without any connections to the street. Both of them have their bases made of concrete and the main structure made of steel with an overall length of about $200 \mathrm{~m}$ and a height of about $30 \mathrm{~m}$ in correspondence to the crown. The activities on the arches were performed during the erection stage of the viaduct, in particular the monitoring activity was carried out from May 2014 to February 2015. The experimental campaign on the real structures has been planned as a consequence of preliminary studies performed at Politecnico di Milano wind tunnel that highlighted wind-induced dynamic instability. In particular, the first two bending modes of the structures showed a critical behaviour and, for this reason, a TMD (Tuned Mass Damping) system was designed in order to control the instability. At first, dynamic tests were performed to check the frequencies, the damping and the modal deflected shape of the first modes of each arch. Both impulsive and harmonic excitations were given [7]. In addition, the excitation given by the surrounding construction site was considered. The tests were then used to tune the three TMDs applied to each arch in correspondence of the antinodes of the two first bending modes, and to check their correct functioning. The two arches were then 
monitored for several months to observe their dynamic behaviour under different wind conditions.

\section{Wind tunnel tests}

Preliminary tests were performed on scaled model of the two arches (geometrical scale 1:40) in the Politecnico di Milano wind tunnel (see Figure 1). One of the two arches was rigid, while the other was completely aeroelastic in order to measure its dynamic response [3].

The vertical acceleration standard deviation measured at a quarter of the span arch is reported in Figure 2 as a function of wind velocity and for different levels of nondimensional damping. The data are referred to the full scale and highlight critical accelerations for low values of structural damping, close to the values expected without the installation of damping devices.

As a consequence of these results, TMDs were designed to control the vibrations relative to the first vertical mode and tests on the real structures were planned.

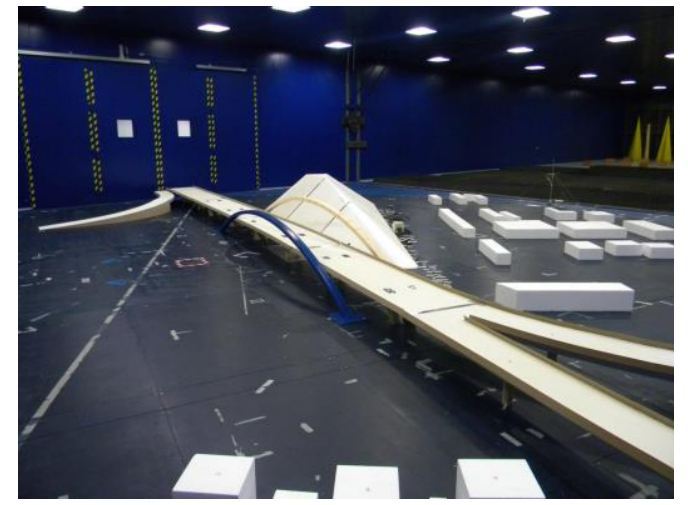

Figure 1. Arches models in the Politecnico di Milano wind tunnel

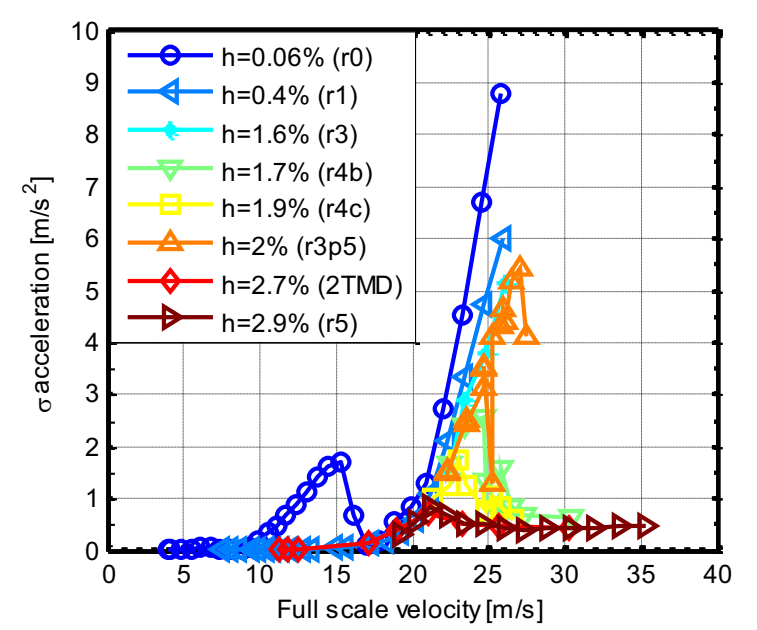

Figure 2. Full scale standard deviation acceleration at $1 / 4$ arch length as a function of full scale wind velocity

\section{Modal identification}

The modal identification of the main characteristics of the two arches were performed with and without TMDs and by using different type of excitation. In particular the tests without the TMD were performed with TMDs installed but blocked in order do not introduce any damping in the system. These tests permit to define the main frequencies of each arch and the structural damping related to the first modes. After releasing the TMD devices the tests were repeated to check the actual damping increase.

Both the arches were instrumented and tested: for brevity's sake only the results relative to one arch are reported, in any case, the other one showed very similar characteristics.

\subsection{Set-up description}

The two arches were instrumented by accelerometers in order to measure the dynamic response to a given excitation. In particular four sections were considered, (see Figure 3) with two accelerometers each in order to measure vertical and horizontal accelerations of the arch. The sections were chosen to have at least one accelerometer close to an antinode position of the first four modes, preliminary defined by means of FEM calculation.

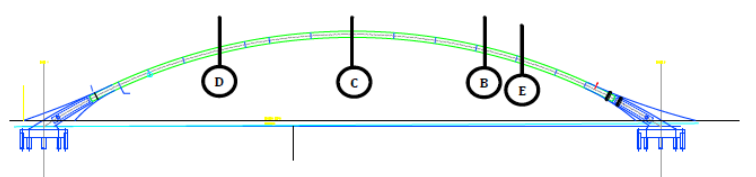

Figure 3. Sketch of the accelerometers placement on the arches

\subsubsection{Impulsive excitation}

Impulsive excitation was given through a static hydraulic actuator able to release up to $100 \mathrm{kN}$ (see Figure $5(\mathrm{sx})$ ). The actuator was connected to the structure by means of a steel cable and a mechanical fuse: the fuse was realized as a test piece made of steel with a breaking load of about $90 \mathrm{kN}$. The actuator was controlled in order to give an increasing load till the desired value, imposing to the structure a static deformation which represent the initial condition to evaluate its free response.

An example of the load time history is reported in Figure 4.

The excitation was given in correspondence of the points $\mathrm{C}$ and $\mathrm{B}$ reported in Figure 3 and both in vertical and horizontal direction.

\subsubsection{Harmonic excitation}

Harmonic excitation was given through a dynamic hydraulic actuator able to release up to $+/-30 \mathrm{kN}$ (see Figure $5(\mathrm{dx}))$. The actuator was connected to the structure through a steel cable and a group of springs, which uncouple the actuator displacement from the structure avoiding modifications in the arch modal shapes. Moving the actuator with a sinusoidal law, a sinusoidal force was produced by means of the springs, this force is applied to the structure and measured by a load transducer. Changing the frequency of the actuator around the natural frequencies of the arch, it was possible 
to define the transfer function between the applied force and the structure displacement $[6,7]$.

The excitation was given in correspondence of the point B reported in Figure 3 in vertical direction.

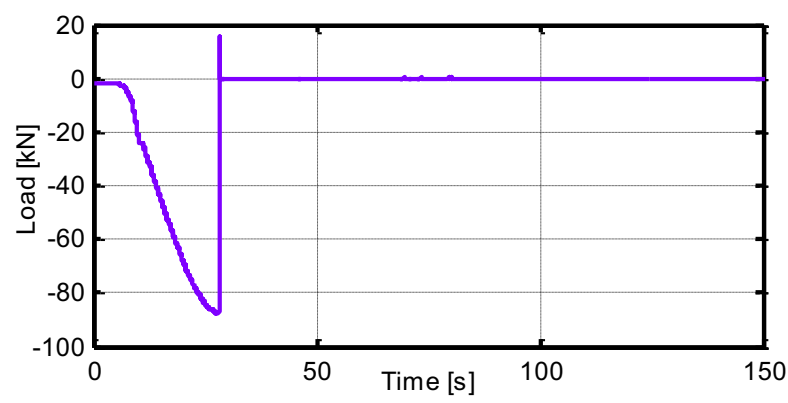

Figure 4. Load applied to the structure during impulsive tests

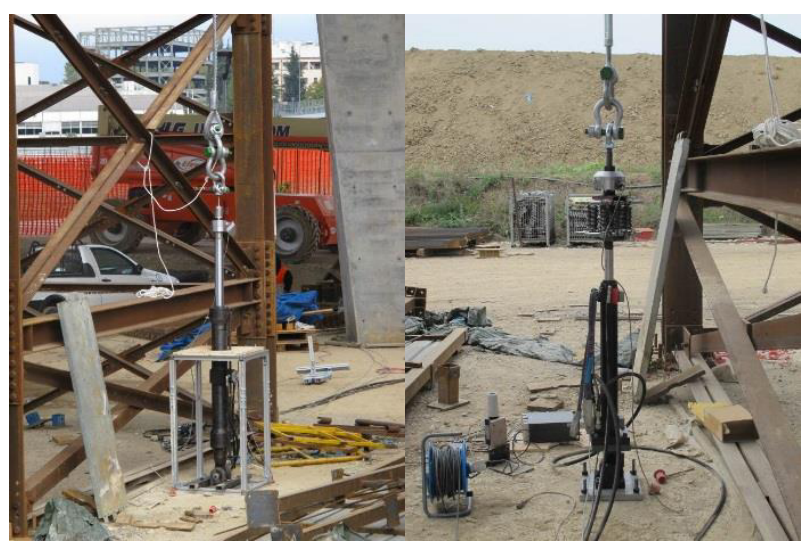

Figure 5. (sx) impulsive excitation $(\mathrm{dx})$ harmonic excitation

\subsection{Results of the modal identification}

\subsubsection{Impulsive excitation}

Impulsive excitation allows to define the frequency and the non-dimensional damping of the first four mode of the structure, two vertical bending modes (Mode 2 and Mode 4) and two horizontal modes (Mode 1 and Mode 3) [8]. The tests were performed without TMD in service (blocked) for all the modes and with TMD operating only for the vertical modes, in order to check their performances. The parameters were obtained considering the free response of the structure after the impulsive excitation given by the actuator.

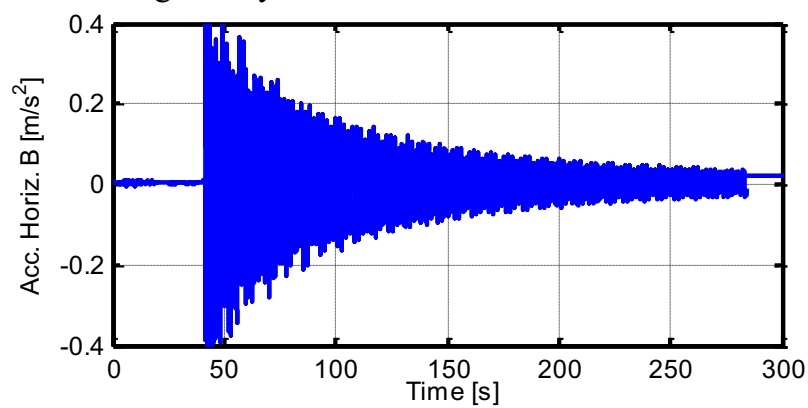

Figure 6. Horizontal decay measured by the accelerometer placed in B (see Figure 3)

An example of the horizontal accelerometer time history measured in point B without TMD is reported in Figure 6. The spectral analysis clearly highlights the harmonic content of the four modes and their frequencies (see Figure 7 for the vertical modes and Figure 8 for the horizontal ones without TMD). The obtained frequencies are very close to the ones estimated numerically by FEM model.

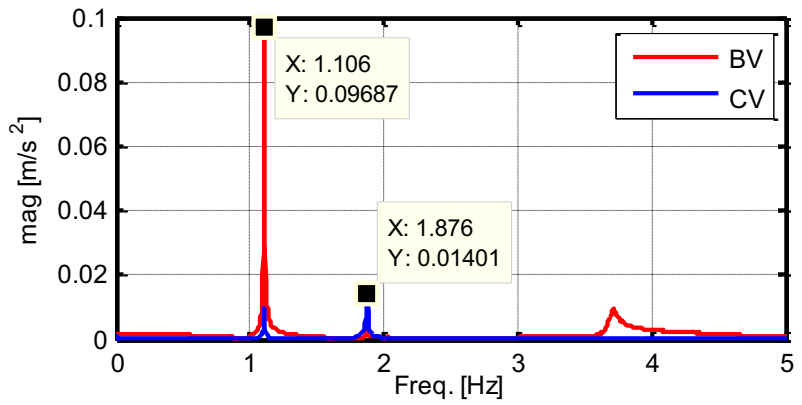

Figure 7. Spectra of the vertical accelerometers placed in $\mathrm{B}$ and in $\mathrm{C}$ (see Figure 3), vertical excitation, without TMD

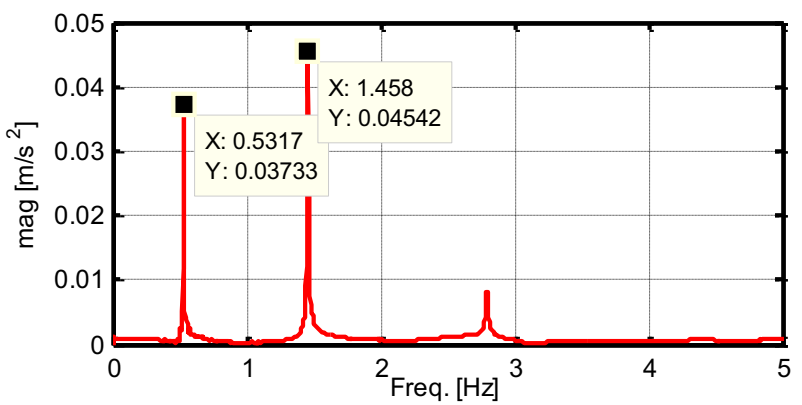

Figure 8. Spectrum of the horizontal accelerometer placed in B (see Figure 3), horizontal excitation

The non-dimensional damping values for the considered modes are obtained applying Hilbert Transform [13] to the accelerometers decay time histories, reported in Figure 9 as a function of the arch displacement in the antinode of each mode (displacements are obtained integrating acceleration signals). It is possible to note that the values are very low, as expected for a slender structure made of steel.

All these results are summarized in Table 1.

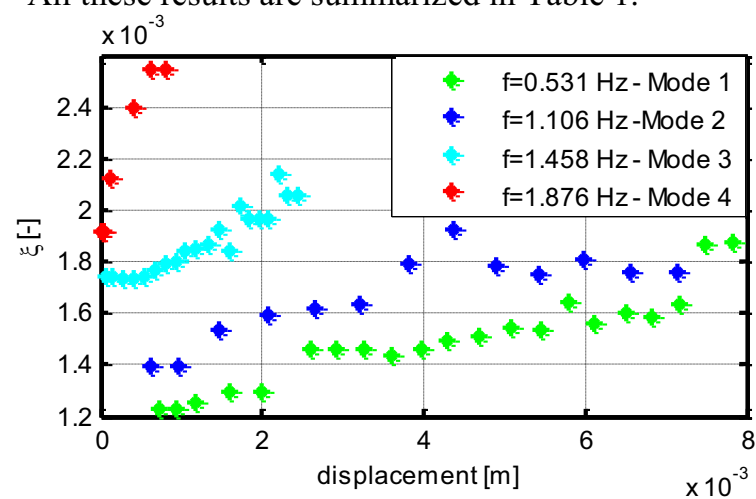

Figure 9. Non-dimensional damping for the first four modes without TMD

Table 1. Main characteristics of the structure

\begin{tabular}{|l|c|c|c|}
\hline & $\begin{array}{c}\text { Frequency } \\
{[\mathrm{Hz}]}\end{array}$ & $\begin{array}{c}\text { Damping without } \\
\text { TMD } \xi[-]\end{array}$ & $\begin{array}{c}\text { Damping with } \\
\text { TMD } \xi[-]\end{array}$ \\
\hline Mode 1 & 0.53 & $0.15 \%$ & \\
\hline Mode 2 & 1.11 & $0.18 \%$ & $5 \%$ \\
\hline Mode 3 & 1.46 & $0.20 \%$ & \\
\hline Mode 4 & 1.88 & $0.23 \%$ & $2 \%$ \\
\hline
\end{tabular}

The vertical excitation tests were repeated after the TMD devices activation. In Figure 10 the spectral 
analysis of vertical acceleration signals are reported: it is possible to note, that the frequencies do not changes significantly but the peaks are characterized by low values and a broader band to indicate a higher damping level. The decays analysis pointed out a strong increase in non-dimensional damping as reported in Figure 11 and in Table 1.

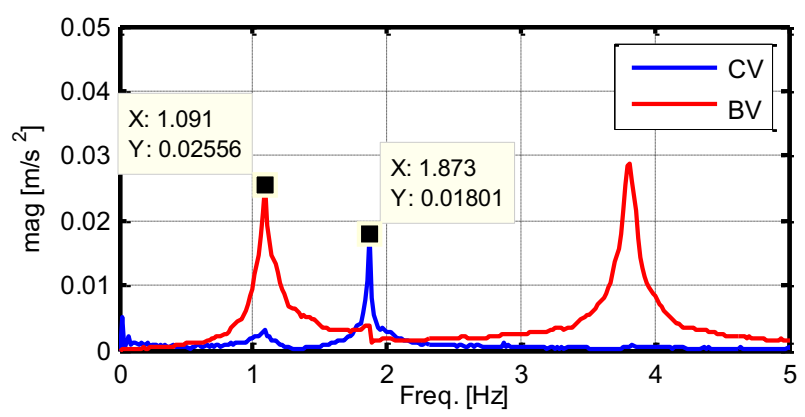

Figure 10. Spectra of the vertical accelerometers placed in B and in C (see Figure 3), vertical excitation, with TMD

The obtained values are comparable with the expected ones in designing TMD devices and they are reached for significant structure oscillation amplitudes. For very low values of arch displacements TMDs are not activated and the damping is close to the structural one.

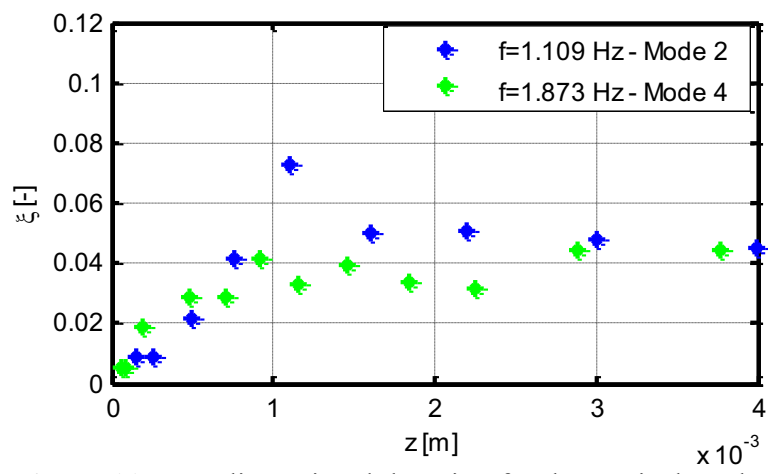

Figure 11. Non-dimensional damping for the vertical modes with TMD

\subsubsection{Harmonic excitation}

Harmonic tests were performed for the two vertical modes in order to confirm the non-dimensional damping values obtained with impulsive, by computing differently the same parameters and relying on data gathered by a different approach excitation for configuration with TMD devices activated. Figure 12 shows magnitude and phase of the transfer function between the excitation force and the structure displacement for the first vertical mode $(\mathrm{f}=1.1 \mathrm{~Hz})$ : force was applied in point $\mathrm{B}$ and the displacement was obtained integrating the acceleration in the same position. Each point of the transfer function was obtained applying a sinusoidal excitation at a specific frequency around the structure natural frequency. As expected, increasing the damping, the transfer function magnitude decreases and the phase changes from $0^{\circ}$ to $180^{\circ}$ less abruptly.

Two different procedure can be used to calculate the non-dimensional damping from the transfer function: phase derivative method and half-power points method.
The phase derivate method permit to define the damping level considering the derivative of the transfer function phase around resonance region. In particular, in resonance condition the following relation is verified:

$$
\left|\frac{\partial \phi(\mathrm{f})}{\partial \mathrm{f}}\right|=\frac{1}{\xi \mathrm{f}_{0}}
$$

Calculating the phase derivative around the resonance region for the TMD configuration a non-dimensional damping equal to $5 \%$ was obtained.

The half-power points method [13] allows to define the damping level considering the frequencies $\mathrm{f}_{1}$ and $\mathrm{f}_{2}$ associated to the half-power points (i.e. the frequencies where the transfer function magnitude is $0.5 \sqrt{2}$ times its maximum peak).
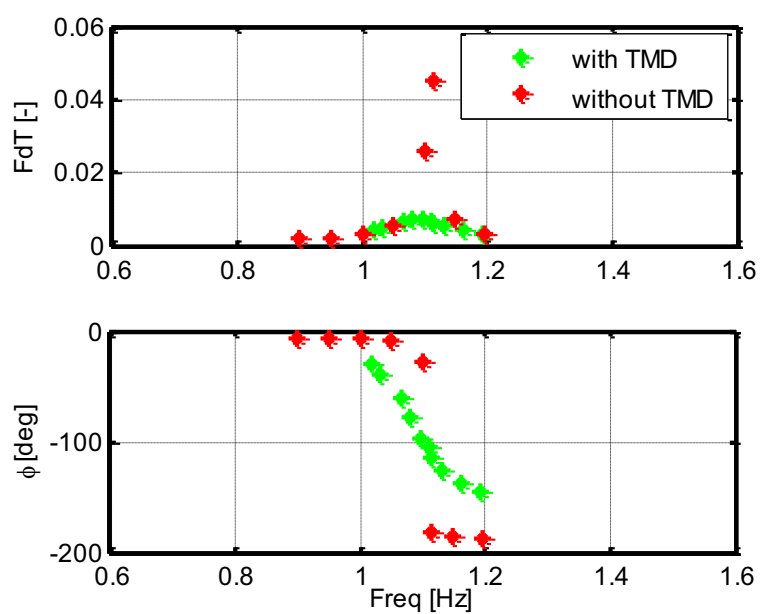

Figure 12. Transfer function between applied load and structure displacement in correspondence of point B (see Figure 3). Both with and without TMD configurations are reported.

The non-dimensional damping can be obtained from the following equation:

$$
\xi=\frac{1}{2}\left(\frac{f_{2}-f_{1}}{f_{0}}\right)
$$

where $f_{0}$ is the natural frequency of the structure $\left(f_{0}=1.1\right.$ $\mathrm{Hz})$.

This method gives a non-dimensional damping equal to $4.4 \%$

\subsubsection{Environmental excitation}

Environmental tests provide structure response to the excitation due to the surrounding (traffic, construction site works, etc). This kind of excitation, although it introduces low power level, permits to identify structure frequencies and modal shapes. The frequencies are in agreement with the ones obtained with the other tests and the first four modal shapes are reported in Figure 13. 

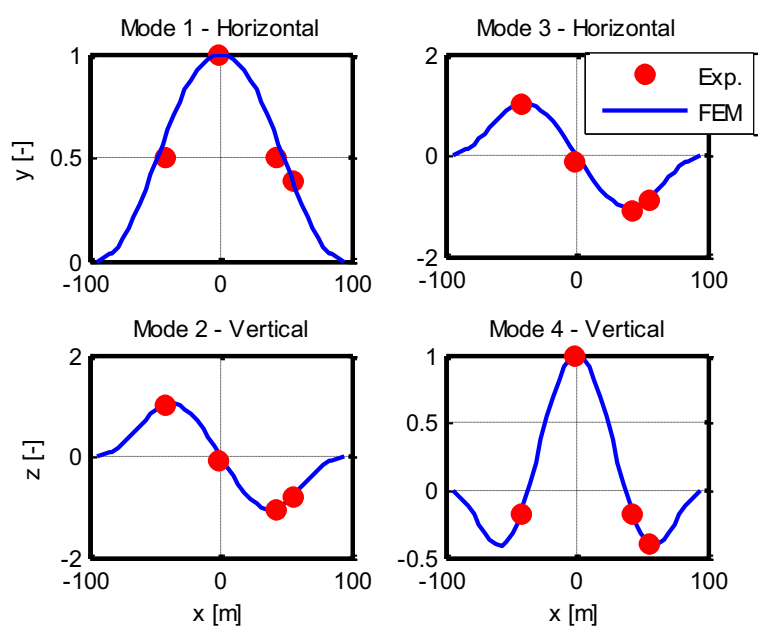

Figure 13. Modal shapes of the first four modes

\section{Monitoring}

Monitoring activity was performed from May 2014 and February 2015 to check the structure behaviour under wind excitation. The activity allows also to verify the TMDs proper functioning over the time. The most significant results obtained from the experimental campaign on the real structures were then compared with the wind tunnel tests results on scaled models.

Both the arches were monitored but for brevity's sake only the results relative to one of them are reported in the paper: in any case the two structure showed very similar behaviour. Moreover, the vertical response of the structure was thoroughly analysed because critical acceleration values were observed in this direction.

\subsection{Set-up description}

During the monitoring campaign the wind velocity in the site was measured, as well as the structures response.

The wind was measured through a sonic anemometer that gives information about velocity and direction. The anemometer was placed on a pole $3.5 \mathrm{~m}$ long on the top of one of the two arches. Figure 14 shows the arches orientation (the two arches are parallel) and the angle definition for the wind direction.

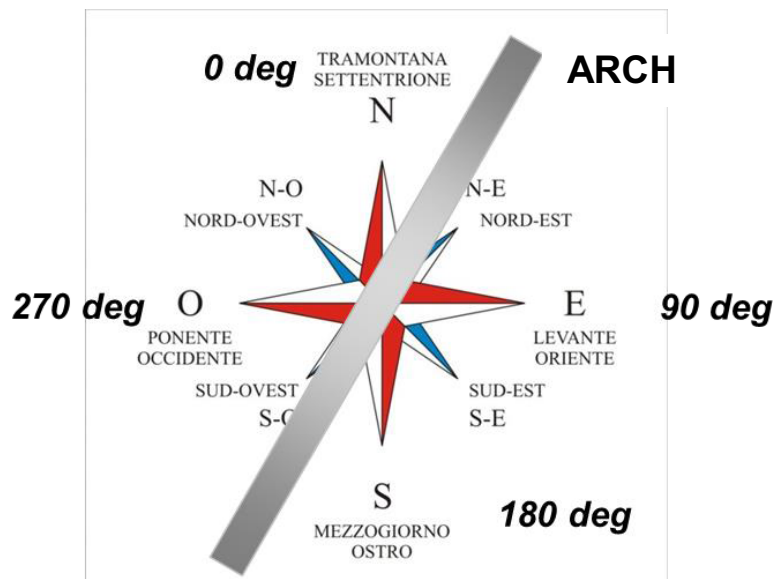

Figure 14. Wind direction definition with respect to the arches
Structures response was measured by a couple of accelerometers for each arch oriented horizontally and vertically (see instrumented section in Figure 15). The chosen section corresponds to the point $\mathrm{E}$ in Figure 3 and it is characterized by non-zero vibration for all the four modes.

Three other accelerometers for each arch were placed on the TMDs in order to check their functioning (see Figure 15).

Moreover, one of two arches was additionally instrumented by strain gauges in four different sections to check local deformations along the time and under strong wind action. In correspondence of the connections between the concrete basis and the main steel arch structure four potentiometers were placed to measure possible undesirable relative displacements.

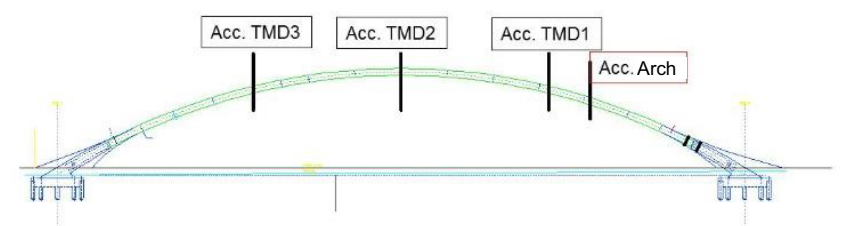

Figure 15. Accelerometers placements on the arches for the monitoring

All the instrumentation signals were stored by devices provided by a processor and able to acquire and process the data in stand-alone mode [12]. The acquisition system was configured in order to acquire data for 10 minutes, to produce synthetic information for each channel (mean value, standard deviation, peak value) and to store them. When high level of wind velocity (mean value higher than $8 \mathrm{~m} / \mathrm{s}$ or peak value higher than $15 \mathrm{~m} / \mathrm{s}$ ) or high level of acceleration (standard deviation higher than $0.1 \mathrm{~m} / \mathrm{s}^{2}$ ) were found, the corresponding complete time history was also stored. The acquisition system was connected to a $3 \mathrm{G}$ net and the data were transferred to a ftp folder on Politecnico di Milano server. This system permits to easily access the data in real time.

\subsection{Results}

Figure 16 shows the synthetic data relative to the wind in terms of mean wind velocity and mean wind direction as function of the time. It is possible to note that only isolate episodes of strong winds were registered and always lower than the design velocity $(23 \mathrm{~m} / \mathrm{s})$ and then the critical wind speed.

Figure 17 permits to visualize wind database (the same data reported in Figure 16), highlighting the directions that happen more frequently and where the strongest winds come from [9]. Dashed lines identify the percentage of data with respect of their total number: the radius of each angular sector is then proportional to the number of sample pertaining the sector. Moreover, each coloured region in a single sector represents a different velocity range and its dimension is proportional to the pertaining number of samples. 

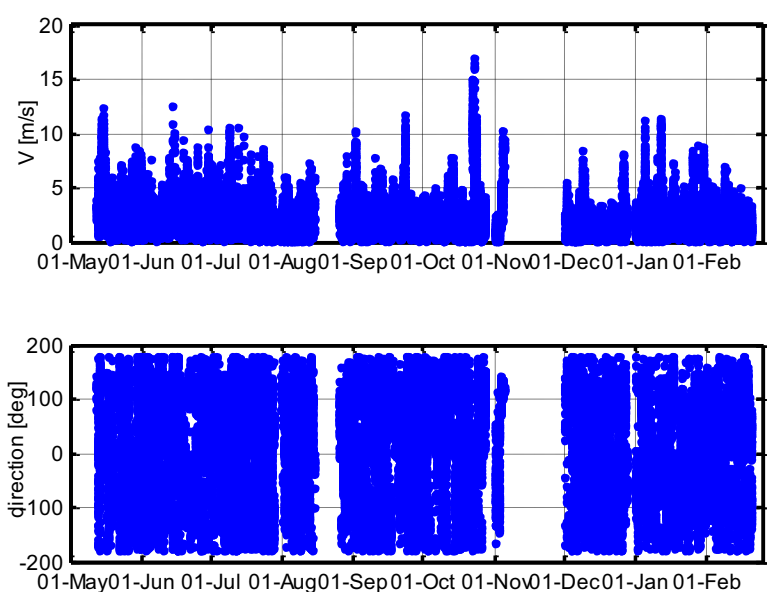

Figure 16. Mean velocity and direction of the wind evaluated on a time basis of 10 minutes
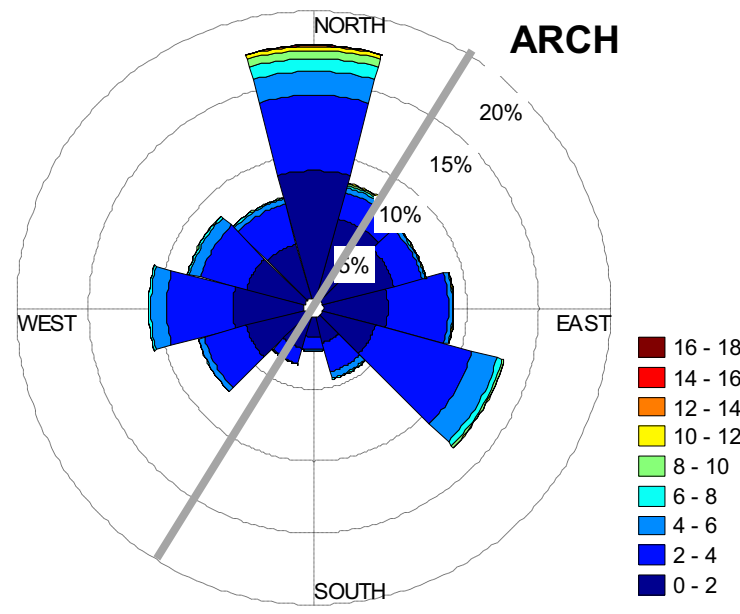

Figure 17. Wind rose: the synthetic data were evaluated on a time basis of 10 minutes

It is possible to note that Northerly winds are the most frequent followed by winds form South/East from West. In terms of wind intensity, Northerly winds are also the strongest followed by winds from East and South/East. Anyway, as stated before, the highest wind velocities are lower than $18 \mathrm{~m} / \mathrm{s}$ and the most frequent winds are lower than $6 \mathrm{~m} / \mathrm{s}$.

Figure 18 shows the vertical response of one of the arches in correspondence of the instrumented section: standard deviations of vertical accelerometer are reported as a function of the time. The data are obtained from the post process directly performed by the acquisition system and so only low pass filter with a high cut off frequency was applied. The figure highlights that generally acceleration are lower than the value considered critical for the structure $\left(0.1 \mathrm{~m} / \mathrm{s}^{2}\right)$ and the isolate episodes that exceed the threshold, analysed more in detail, showed instantaneous peaks due to environmental excitation, typically related to the construction site works. A detailed analysis shows, in addition, that the acceleration level during the day is higher than during the night: this can be explained considering that during the day both the wind excitation and the environmental excitation are higher.

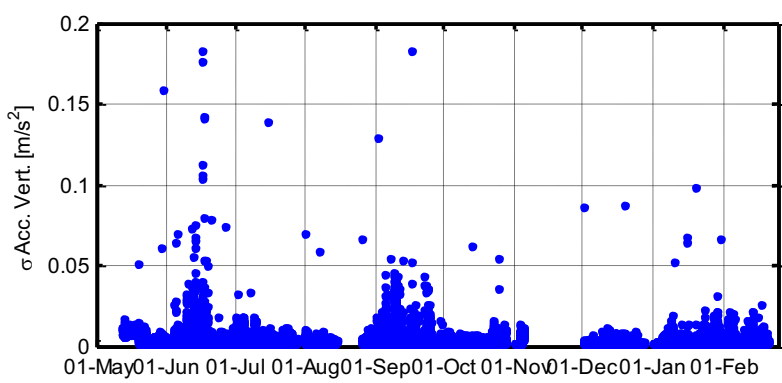

Figure 18. Standard deviation of the vertical accelerometer evaluated on a time basis of 10 minutes

Figure 19 shows, as an example, two time histories of the vertical accelerometer registered in different moment of a day. The up time history shows the typical trend related to environmental excitation with isolated build up and decay, on the other hand the down time history shows a more distributed excitation during the time related to wind action.
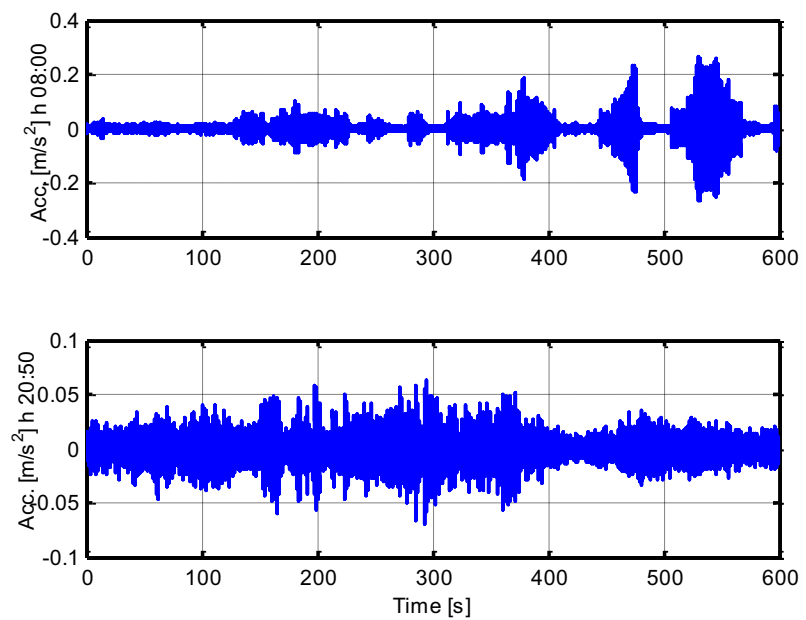

Figure 19. Time histories of vertical accelerometer in two different moments of the day

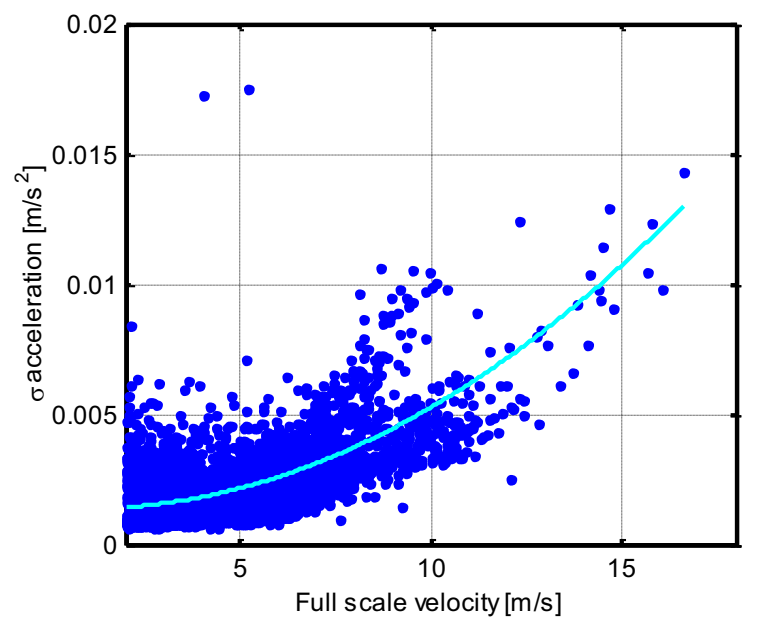

Figure 20. Standard deviation of the vertical accelerometer (low pass filter $10 \mathrm{~Hz}$ ) as a function of the wind velocity

Figure 20 shows the vertical accelerometer standard deviation obtained from the stored time histories (one file per hour and one file when threshold values are exceeded) plotted as a function of the wind velocity. The signal was filtered with a low pass filter at $10 \mathrm{~Hz}$ in order 
to highlight only the contributions related to the first natural frequencies, the ones excited by the wind. It is possible to note a parabolic trend of the standard deviation with the wind velocity, the values that diverge from the trend are related to environmental excitation phenomena.

Data of Figure 20 are then divided for angular section of wind direction and each group of data is interpolated with a parabolic curve: some curves are reported in Figure 21. It is possible to note that the higher wind velocities were measured for sector $-15^{\circ} / 15^{\circ}$ that includes also the most frequent winds (see Figure 17). However, this sector cannot be considered critical because the derivative of the curve is quite low which means that the accelerations grows slowly with the wind velocity: curve derivative can be considered indicative of the wind capability to excite the structure. On the contrary, sectors $75^{\circ} / 105^{\circ}$ and $105^{\circ} / 135^{\circ}$ are potentially critical due to the curves derivatives: if a very strong wind may occur from these directions it can become problematic for the structure. In fact, these wind flows are almost perpendicular to the arch axis, so that they can excite more significantly the structure with respect to the winds that flow along the arch. Fortunately, as suggested by Figure 17, the winds coming from these directions are generally lower than $6 \mathrm{~m} / \mathrm{s}$. Similar trend is observed for sector $-75^{\circ} /-45^{\circ}$, placed in opposite direction while sector $-45^{\circ} /-15^{\circ}$ is similar to the Northerly one.

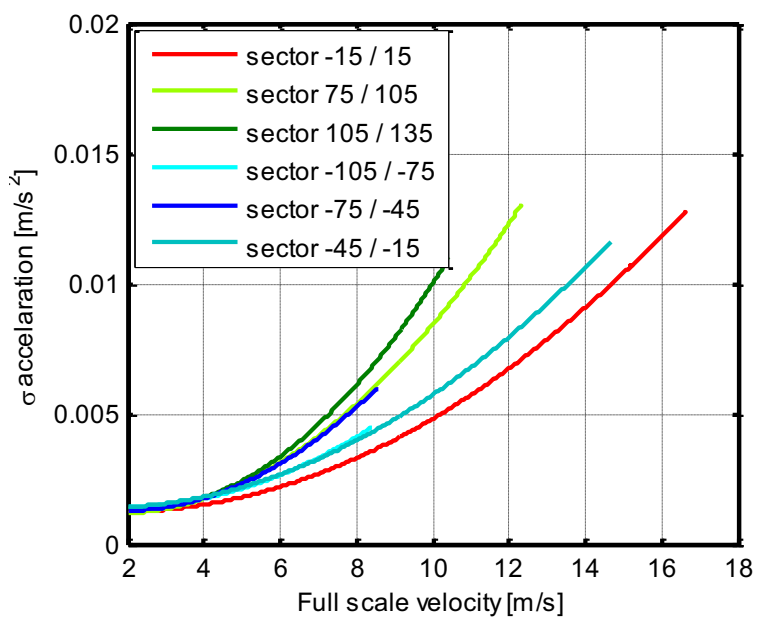

Figure 21. Parabolic interpolation of vertical acceleration standard deviations as a function of wind direction and wind velocity

Some full scale results are compared with three different exposures tested in the wind tunnel (Figure 22): a very good agreement between the data can be observed (the wind tunnel data were properly scaled). Further comparison is reported in Figure 23 where standard deviations measured in a windy day are compared with wind tunnel test performed at different level of nondimensional damping. Full scale data are in agreement with the ones related to the higher non-dimensional damping.

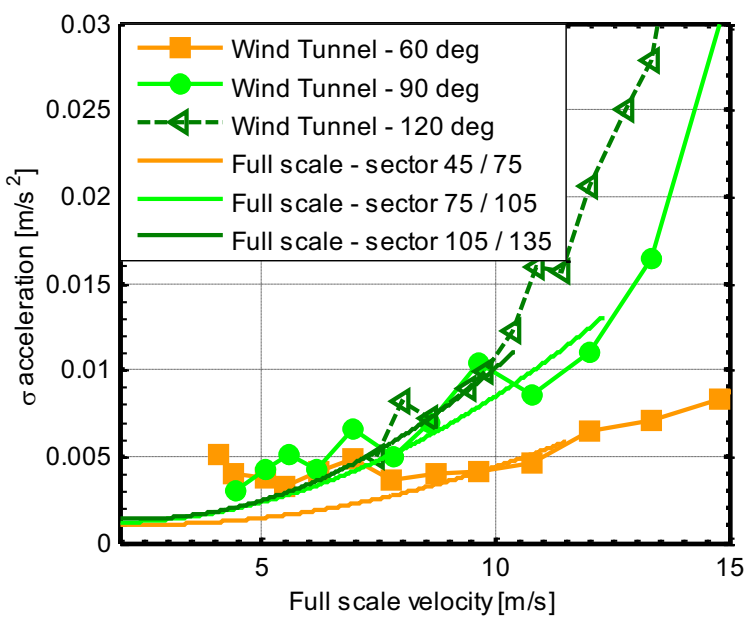

Figure 22. Comparison between full scale and wind tunnel results

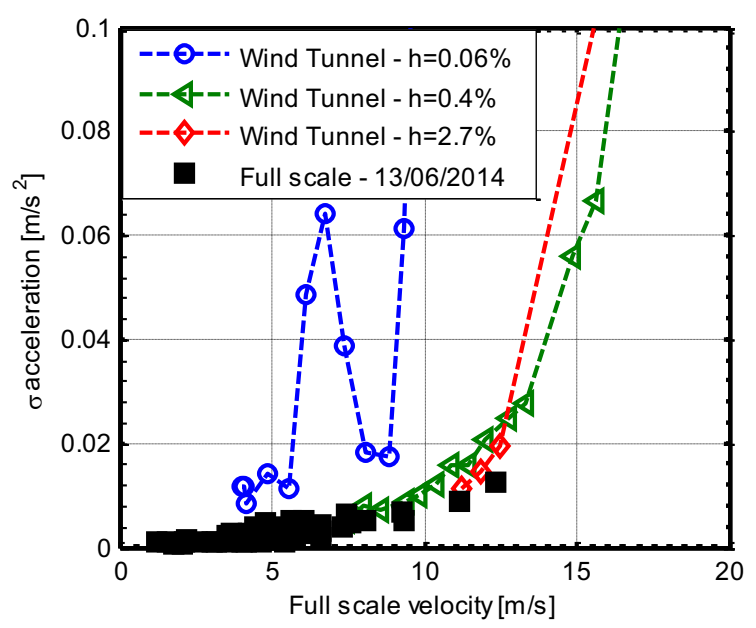

Figure 23. Comparison between full scale and wind tunnel results

\section{Conclusion}

Modal parameters identification of the two arches built in the new area of Expo 2015 in Milan was performed by using different excitation techniques: all the experimental estimations of frequencies and mode shapes are in good agreement each other and with the numerical data obtained from FEM model. The numerical model used for the structures design can be considered validated. The different techniques used to identify the non-dimensional damping gave comparable results, in particular for the configuration with activated TMDs a value of about $4 \%$ was found. This level of damping exceeds the expected values in design phase, confirming the good functioning of TMD system.

Moreover, the continuous monitoring highlighted the good agreement between full scale and wind tunnel response of the structures: this allows to extrapolate the dynamic response of the real structure under strong wind condition, with limited oscillation amplitudes as well as observed during wind tunnel tests.

That means that the structure can be considered safe from an aerodynamic point of view. 
However, the monitoring activity is continuing in order to check if in the site wind velocities higher than the measured ones may occur.

\section{References}

1. E. Simiu and H.R Scanlan, Wind Effects on Structures (John Wiley \& Sons, New York, 1996).

2. S. O. Hansen and C. Dyrbye, Wind loads on structures, (Chichester, 1996).

3. M. Belloli, F. Fossati, S. Giappino, S. Muggiasca and M. Villani, 'On the aerodynamic and aeroelastic response of a bridge tower' J. Wind Eng. Ind. Aerodyn. 99 (2011) 729-733

4. J. P. Den Hartog, Mechanical vibrations, (Dover, 1985)

5. J. M. W. Brownjohn, E. P. Carden, C. R. Goddard and G. Oudin, 'Real-time performance monitoring of tuned mass damper system for a $183 \mathrm{~m}$ reinforced concrete chimney', Journal of Wind Engineering and Industrial Aerodynamics, 98 (2010): 169-179.

6. T Argentini, L. Rosa and A. Zasso, 'Experimental evaluation of Hovenring bridge stay-cable vibration', WIT Transactions on Modelling and Simulation, 2013, 427-437.

7. T Argentini, M. Belloli, L. Rosa, E. Sabbioni and A. Zasso 'Modal identification of stays and deck of a cable-stayed bridge, Proceedings of the International Conference on Noise and Vibration Engineering, ISMA 2012, Leuven, Belgium, pp. 1031--1043.

8. T. Argentini et al., 'Modal identification of a cablestayed bridge by means of truck induced vibrations', Conference Proceedings of the Society for Experimental Mechanics Series, Jacksonville, Florida, pp. 165-172.

9. N. Martins, E. Caetano, S. Diord, F. Magalhães and A. Cunha, 'Dynamic monitoring of a stadium suspension roof: Wind and temperature influence on modal parameters and structural response', Engineering Structures 59 (2014), 80-94

10. F. Magalhães, A. Cunha, and E. Caetano,' Vibration based structural health monitoring of an arch bridge: From automated OMA to damage detection', Mechanical Systems and Signal Processing 28 (2012), 212-228

11. W. Hu, E. Caetano and A. Cunha, 'Structural health monitoring of a stress-ribbon footbridge', Engineering Structures 57 (2013), 578-593

12. I. Bayati, M. Belloli, L. Rosa and A. Zasso 'Full scale monitoring of the twin chimneys of the Rovinari power plant, Proceeding of EVACES, 1921 October, 2015, Dübendorf, Switzerland.

13. J. S. Bendat and A. G. Piersol, 'Random Data: Analysis and Measurement Procedures' (John Wiley \& Sons, New York, 1966). 